\title{
Asymmetric Synthesis of Homoallylic Alcohols featuring Vicinal Tetrasubstituted Carbon Centers via Dual Pd/Photoredox Catalysis
}

\author{
Sijing Xue, ${ }^{\dagger}$ Bart Limburg, ${ }^{\dagger *}$ Debasish Ghorai, ${ }^{\dagger}$ Jordi Benet-Buchholz ${ }^{\dagger}$ and Arjan W Kleij ${ }^{\dagger, \xi *}$ \\ ${ }^{+}$Institute of Chemical Research of Catalonia (ICIQ), the Barcelona Institute of Science and Technology, Av. Països \\ Catalans 16, 43007 - Tarragona, Spain. E-mail: akleii@iciq.es, blimburg@iciq.es \\ ${ }^{\S}$ Catalan Institute of Research and Advanced Studies (ICREA), Pg. Lluís Companys 23, 08010 Barcelona, Spain \\ KEYWORDS: asymmetric synthesis, homoallylic alcohols, palladium, photoredox, quaternary carbons
}

\begin{abstract}
Dual palladium/photoredox-catalysis provides an effective method for the asymmetric synthesis of vicinal $\alpha, \beta$-tri/tetra- or $\alpha, \beta$-tetra-substituted homoallylic alcohols. Regio- and enantioselective decarboxylative allylic alkylation of vinyl cyclic carbonates is reported using Hantzsch type esters as radical precursors. The developed methodology combines the use of versatile and accessible reagents and can be operated under mild reaction conditions giving the target molecules in appreciable to good yields, high branch-selectivity and appreciable enantiomeric ratios of up to 94:6. This protocol marks a rare example of the use of prochiral electrophiles for the creation of vicinal congested carbon centers
\end{abstract}

Introduction: Chiral quaternary carbon centers are ubiquitous in natural products and pharmaceuticals, ${ }^{1}$ but still present chemical challenges in their synthesis. While significant progress has been made over the recent decades in the asymmetric construction of quaternary carbon stereocenters, ${ }^{2}$ the enantioselective formation of acyclic vicinal tetrasubstituted carbon centers represents a more daunting challenge. ${ }^{1 a+b, 3}$ Among the strategies that have proven to be both versatile and effective, asymmetric allylic alkylation (AAA) represents one of the most powerful methods for the construction of carbon-carbon bonds resulting in quaternary carbon stereocenters. ${ }^{2 a-b, g, 4}$ Two main AAA approaches are known that exploit stereocontrol over either prochiral nucleophiles ${ }^{5}$ or prochiral electrophiles. ${ }^{6}$ Although various efficient metal-assisted protocols have been established, to date the enantioselective preparation of organic target molecules comprising acyclic vicinal tetrasubstituted carbon centers utilizing prochiral electrophiles remains underexplored (Scheme 1a).

Pd-catalyzed asymmetric allylic substitution reactions of vinyl epoxides ${ }^{7}$ and vinyl cyclic carbonates (VCCs) ${ }^{8}$ serving as prochiral electrophilic allylic surrogates have been recently reported as efficient approaches to forge compounds featuring a tetrasubstituted carbon stereocenter. While only a handful of studies report the efficient use of olefin-substituted VCCs in allylic alkylation (Scheme $\left.1 b, R^{2}-R^{4} \neq H\right)^{9}$ and more particularly towards branched allylic products, ${ }^{9 h, i}$ as far as we are aware only two reports exist that briefly discuss the use of more elaborate carbonate ring substituted congeners (Scheme $1 b, R^{5}$ and $R^{6} \neq H$ ). ${ }^{9 f, g}$ The successful conversion of these latter types of congested substrates in transformations leading to compounds having vicinal tetrasubstituted carbon 
centers would greatly expand the application potential of VCCs and advance the synthesis of otherwise elusive carbon stereocenters.

(a) Vicinal/Acyclic Quaternary Carbon Centers using Prochiral Electrophiles:

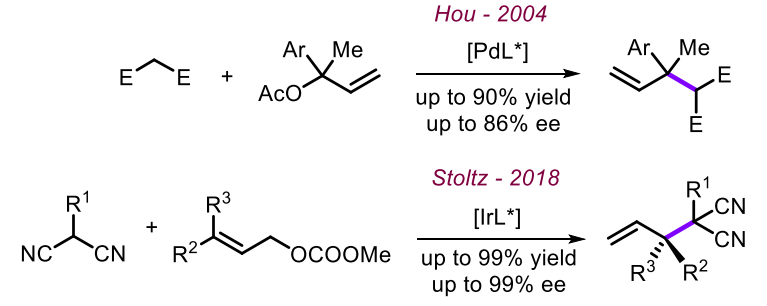

(b) Progress with VCCs in (Asymmetric) Allylic Alkylation:

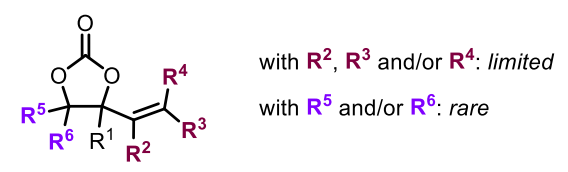

(c) THIS WORK: AAA of VCCs towards Vicinal Tetrasubstituted Carbon Centers

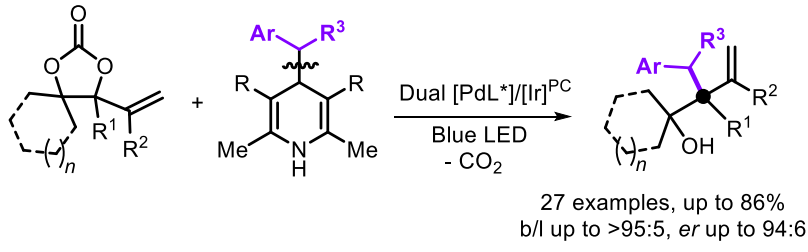

Scheme 1. (a) Limited Progress with Prochiral Electrophiles, (b) Limitations in the use of VCCs (b) and (c) Current Approach towards Elusive Stereocenters using AAA Strategies.

In order to expedite the potential of these challenging functional substrates in allylic alkylation processes, we envisioned that the use of dual transition metal/photoredox catalysis ${ }^{10}$ could offer an alternative yet powerful approach that could circumvent the limitations encountered in classical catalytic allylic substitution reactions (cf., Scheme 1b). Herein, we report the combination of photoredox and Pd-catalyzed AAA of vinyl cyclic carbonates using Hantzsch type esters as radical precursors ${ }^{11}$ affording homoallylic alcohol products with either $\alpha, \beta$-tri/tetraor $\alpha, \beta$-tetra-substituted carbon centers (Scheme 1c). The developed protocol combines mild reaction conditions, avoids the use of stoichiometric organometallic reagents and produces a series of products with acyclic vicinal tetrasubstituted carbon centers in good yields and high regio- and enantiocontrol. 


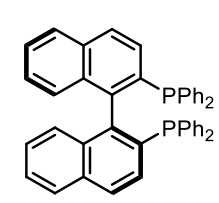

L1: (S)-BINAP

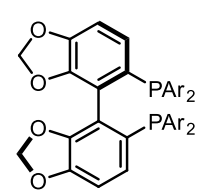

L2: $\mathrm{X}=\mathrm{Y}=\mathrm{H}:(R)$-SegPhos

L3: $\mathrm{X}=\mathrm{Me}, \mathrm{Y}=\mathrm{H}:(R)-\mathrm{DM}$-SegPhos

L4: $\mathrm{X}=t \mathrm{Bu}, \mathrm{Y}=\mathrm{OMe}:(R)$-DTBM-SegPhos
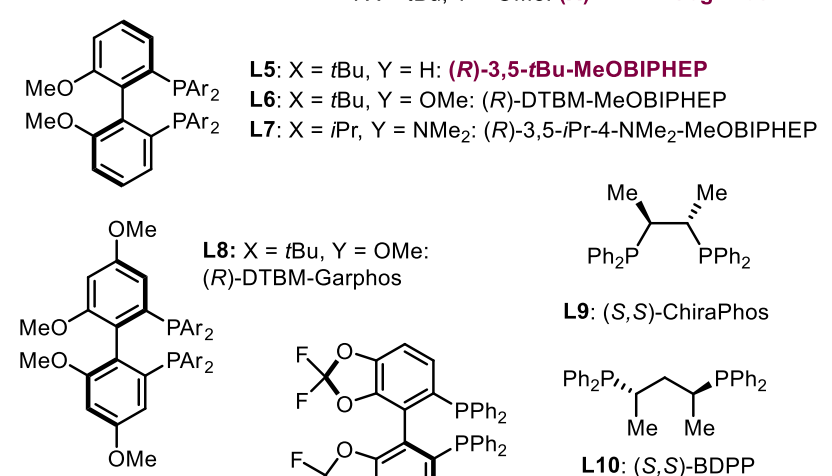

8: $X=t B u, Y=O M e$ $(R)$-DTBM-Garphos

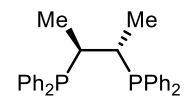

L9: $(S, S)$-ChiraPhos
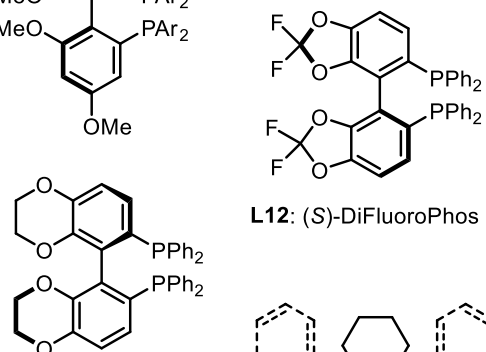

L12: (S)-DiFluoroPhos

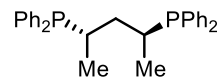

L10: $(S, S)$-BDPP

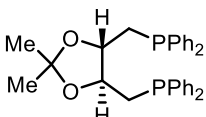

L11:

$(S, S)-(+)$-DIOP

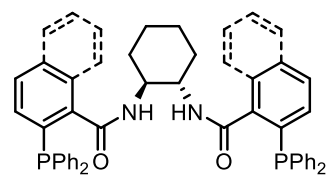

L14: (S,S)-Dach-phenyl, L15: (S,S)-Dach-naphthyl

Figure 1. Phosphine-based ligands of Table 1.

Results and discussion: We started our investigation by using VCC 1a and substituted Hantzsch ester $\mathbf{2 a}$ and examining the envisioned coupling while screening a variety of chiral ligands, Ir-based photocatalysts, solvents and base additives (Supporting Information, SI: Tables S1-S5). The most relevant selection of these data is provided in Table 1. After determining the most effective photocatalyst $\left(P C, \operatorname{lr}(p p y)_{2}(d t b b p y) P F_{6}\right)$, type of base $\left(\mathrm{Cs}_{2} \mathrm{CO}_{3}\right)$ and solvent (acetonitrile), a wide variety of chiral diphosphine ligands (see Figure 1 and $\mathrm{SI}$ ) were scrutinized in the benchmark protocol.

The use of BINAP as a chiral ligand (entry 1) did not lead to any asymmetric induction and 3aa was produced in a low yield (25\%). The utilization of Segphos type diphosphines proved to be more productive (entries 2-4), and variation of the P-aryl groups gave 3aa in 60\% yield with excellent regio- (b:I >95:5) and appreciable enantioselectivity (er $=86: 14)$. Structurally related BIPHEP and Garphos ligands (entries 4-8) also provided good results, with the use of $\mathbf{L} \mathbf{5}$ being most efficient (entry 5: 71\%, b:I >95:5, er $=89: 11$ ). 
Table 1. Part of the Screening Data towards the Synthesis of Homoallylic Alcohol 3aa. ${ }^{a}$

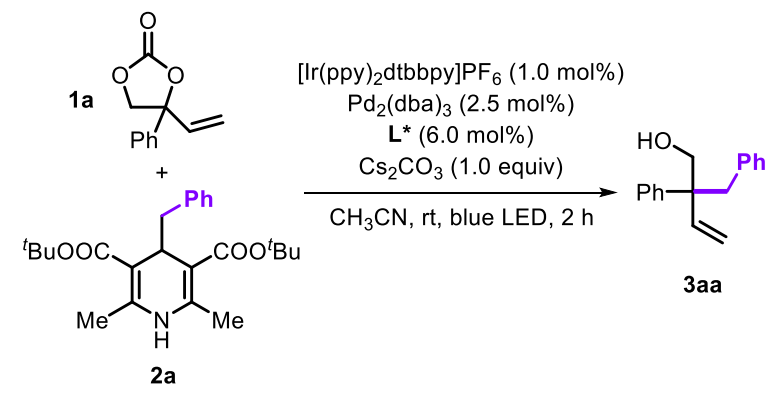

\begin{tabular}{|c|c|c|c|c|}
\hline entry & $\mathbf{L}^{*}$ & yield $(\%)^{a}$ & $b / l^{a}$ & $e r^{a}$ \\
\hline 1 & L1 & 25 & $65: 35$ & 0 \\
\hline 2 & L2 & 26 & $64: 36$ & $64: 36$ \\
\hline 3 & L3 & 31 & $70: 30$ & $63: 37$ \\
\hline 4 & L4 & 60 & $>95: 5$ & $86: 14$ \\
\hline 5 & L5 & 71 & $>95: 5$ & $89: 11$ \\
\hline 6 & L6 & 66 & $>95: 5$ & $84: 16$ \\
\hline 7 & L7 & 65 & $>95: 5$ & $86: 14$ \\
\hline 8 & L8 & 60 & $93: 7$ & $85: 15$ \\
\hline 9 & L9 & 12 & $22: 78$ & - \\
\hline 10 & L10 & 16 & $31: 69$ & $50.5: 40.5$ \\
\hline 11 & L11 & 12 & $28: 72$ & - \\
\hline 12 & L12 & 28 & $73: 26$ & $40: 60$ \\
\hline 13 & L13 & 30 & $61: 39$ & $63: 37$ \\
\hline 14 & L14 & 11 & $27: 73$ & - \\
\hline 15 & L15 & 12 & $22: 78$ & - \\
\hline $16^{b}$ & L4 & 65 & $>95: 5$ & $87.5: 12.5$ \\
\hline $17^{c}$ & L4 & 25 & 61:39 & $87.5: 12.5$ \\
\hline $18^{d}$ & L4 & 0 & - & - \\
\hline $19^{e}$ & L4 & 71 & $>95: 5$ & $88: 12$ \\
\hline $20^{e}$ & L5 & 75 & $>95: 5$ & $89: 11$ \\
\hline
\end{tabular}

${ }^{a}$ 1a $(0.10 \mathrm{mmol}), 2 \mathrm{2a}(0.15 \mathrm{mmol}), \mathrm{Ir}(\mathrm{ppy})_{2}(\mathrm{dtbbpy}) \mathrm{PF}_{6}(1.0 \mathrm{~mol} \%), \mathrm{Pd}_{2}(\mathrm{dba})_{3}(2.5 \mathrm{~mol} \%), \mathrm{L}^{*}(6.0 \mathrm{~mol} \%), \mathrm{Cs}_{2} \mathrm{CO}_{3}(0.10 \mathrm{mmol})$ were combined in $\mathrm{CH}_{3} \mathrm{CN}(2.0 \mathrm{~mL})$ at $25^{\circ} \mathrm{C}$ under blue LED radiation $(445 \mathrm{~nm}, 0.7 \mathrm{~A}$, corresponding to a photon flux of $1.2 \mu$ einstein/s) for $2 \mathrm{~h}$. Yields and $\mathrm{b} / \mathrm{I}$ ratios were determined by ${ }^{1} \mathrm{H}$ NMR analysis using $\mathrm{CH}_{2} \mathrm{Br}_{2}$ as an internal standard. Enantiomeric ratios (er) were determined by UPC2. ${ }^{b}$ In the absence of $\mathrm{Cs}_{2} \mathrm{CO}_{3} .{ }^{9}$ In the absence of $\operatorname{Ir}(\mathrm{ppy})_{2}$ (dtbbpy)PF ${ }_{6}{ }^{d}$ In the dark. ${ }^{e}$ In the absence of $\mathrm{Cs}_{2} \mathrm{CO}_{3}$, under blue LED radiation (445 $\mathrm{nm}$, 1.0 A, corresponding to a photon flux of $1.6 \mu$ einstein/s). 
The use of other diphosphine ligands (L9-L15) did not improve the process outcome (entries 9-15). Further variations of the protocol (entries 16-19) were first carried out with (R)-DTBM-SegPhos being cheaper than $(R)$ 3,5-tBu-MeOBIPHEP, but providing nearly the same regio- and enantiocontrol (cf., entries 4 versus 5 ). In the absence of $\mathrm{Cs}_{2} \mathrm{CO}_{3}$, 3aa was obtained in a slightly higher yield and er (entry 16 versus 4), and therefore was omitted for the optimized conditions (vide infra). Omitting the iridium PC (entry 17) gave 3aa in $25 \%$ yield suggesting that Hantzsch ester $2 \mathrm{a}$ could itself also serve as a photoreductant to form a radical cation that subsequently generates, through homolytic cleavage, the requisite alkyl radical for the $\mathrm{C}-\mathrm{C}$ coupling. ${ }^{12}$ As expected, no product was detected without blue LED irradiation, but by further increasing the light intensity, the yield of 3aa increased to 71\% (entry 19). With these alternative conditions in hand, we then re-used (R)-3,5-tBu-MeOBIPHEP L5 as ligand, which afforded the product in 75\% yield and with an er of 89:11 (entry 20 ).

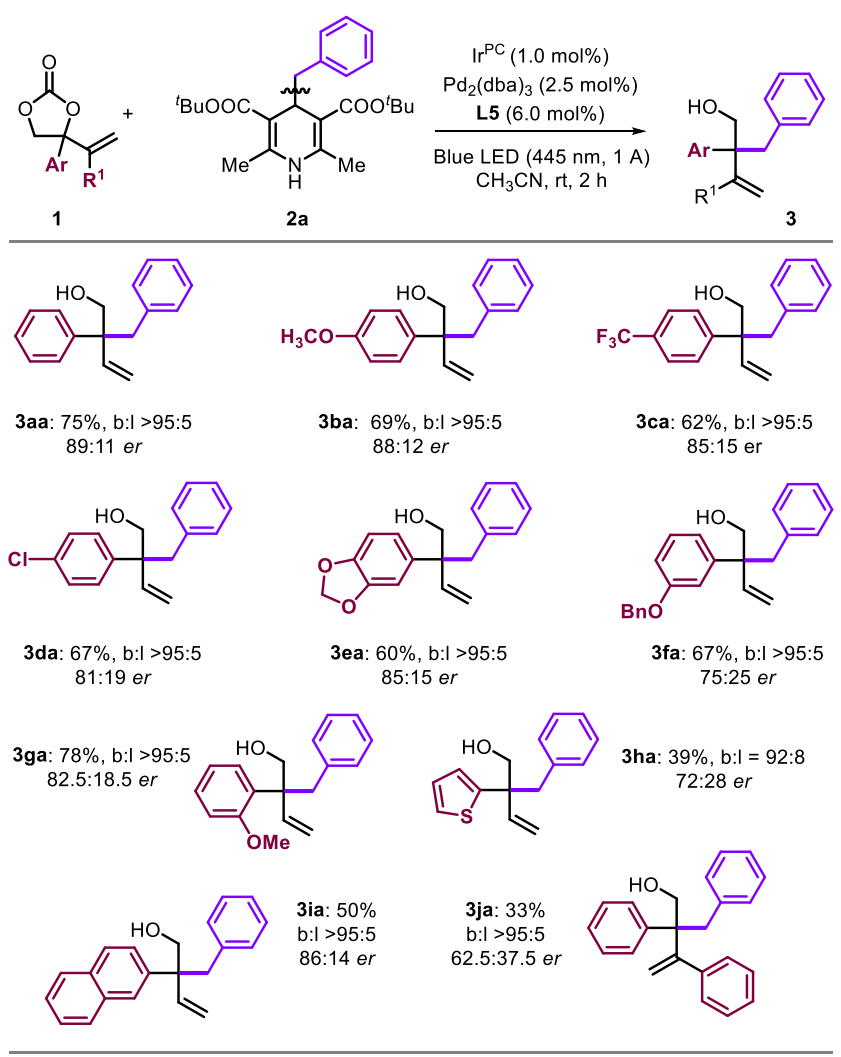

Scheme 2. Product scope using various VCCs to generate quaternary carbon stereocenters. Reaction conditions: 1 (0.10 mmol), 2 a (0.15

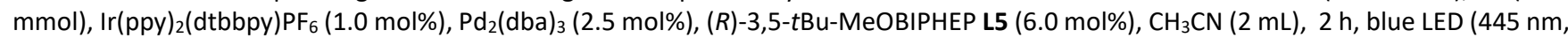
$1 \mathrm{~A}$, corresponding to a photon flux of $1.6 \mu$ einstein/s). Yields of the isolated, column-purified products are reported. The enantiomeric ratios (er values) were determined by UPC2. The b/I ratios were determined by ${ }^{1} \mathrm{H}$ NMR analysis.

With these optimized conditions we then examined the generality of similar substrate combinations providing homoallylic alcohols with quaternary carbon stereocenters (Scheme 2, 3aa-3ja). Variation of the aryl substituents on the VCC in the presence of Hantzsch ester 2a generally provided the homoallylic alcohols with remarkable branch-selectivity (b:I >95:5) and in appreciable isolated yields of up to 78\% (3ga). Good enantio-induction levels of up to 89:11 er for the majority of the products were achieved except for $\mathbf{3 f a}$, 3ha and $\mathbf{3 j a}$. Whereas for $\mathbf{3 h a}$ the presence of the thiophen-2-yl group could interfere through coordination to Pd(allyl) intermediates, the use of a 
VCC with an additional substituent $\left(R^{1}=P h, 1 j\right)$ on the vinyl group hence increasing the steric demand substrate activation was detrimental to both the product yield (33\%) and optical purity (62.5:37.5 er).

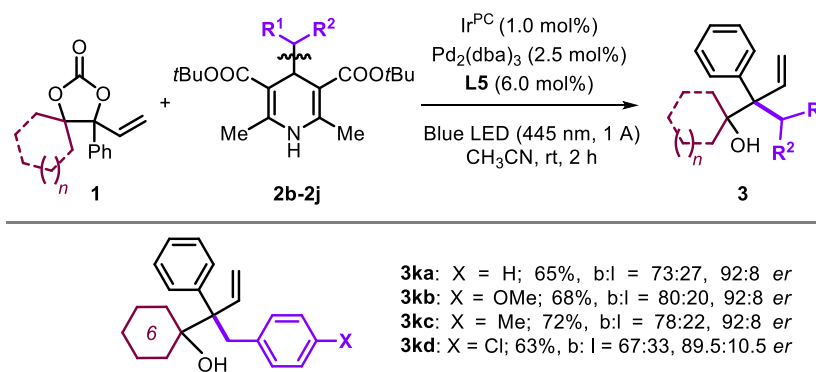

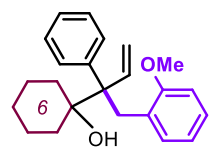

3ke: $58 \%, b: l=78: 22$ $92.5: 7.5 \mathrm{er}$

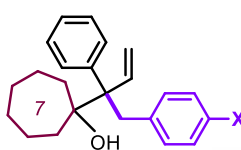
$\mathrm{b}: l=62: 38,92: 8$ er 3lb: $\mathrm{X}=\mathrm{OMe} ; 65 \%$ $\mathrm{b}: \mathrm{l}=75: 25,92: 8 \mathrm{er}$
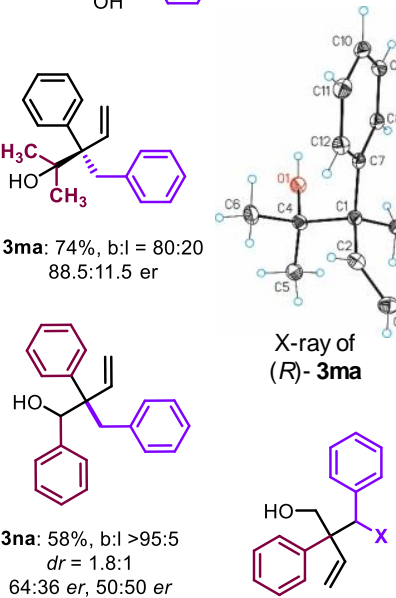

3ma: $74 \%, b: l=80: 20$ $88.5: 11.5$ er

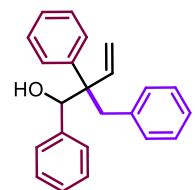

3na: $58 \%$, b:l $>95: 5$

$$
d r=1.8: 1
$$

$64: 36$ er, $50: 50$ er $92: 8$ er

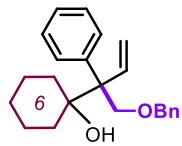

3kg: $48 \%, b: I=67: 33$ 90:10 er

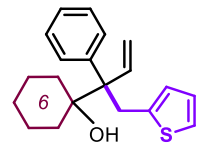

3kf: $67 \%$, b:I $=85: 15$

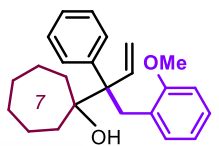

3le: $55 \%, b: l=66: 34,94: 6$ er
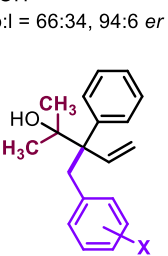

3mb: $\mathrm{X}=p$-OMe, $86 \%$

$\mathrm{b}: l=87: 13,89.5: 10.5 \mathrm{er}^{b}$

3me: $\mathrm{X}=0-\mathrm{MeO}, 56 \%$

$\mathrm{b}: \mathrm{l}=83: 1789: 11 \mathrm{er} \mathrm{r}^{\mathrm{b}}$

3ah: $X=P h ; 57 \%, b: l=92: 8,89.5: 10.5 e r^{c}$

3ai: $\mathrm{X}=\mathrm{Me} ; 53 \%, \mathrm{~b}: \mathrm{I}>95: 5, d r=1.0$ $88.5: 11.5 \mathrm{er}, 89: 11 \mathrm{er}$

3aj: X = Et; $66 \%$, b:l >95:5, $d r=1.0$ 90.5:9.5 er, 90.5:9.5 er

Scheme 3. Product scope using various VCCs to generate chiral homoallylic alcohols having vicinal tetrasubstituted carbon centers. Reaction conditions are the same as in Scheme 2. Yields of the isolated, column-purified products are reported. The enantiomeric ratios (er values) were determined by UPC2. The b/I ratios were determined by ${ }^{1} \mathrm{H}$ NMR analysis. ${ }^{b}$ Reaction time was $4 \mathrm{~h}$. ${ }^{c}$ The corresponding Hantzsch nitrile was used.

In a second, more dedicated embodiment of the substrate scope we primarily selected VCCs with further substitution on the carbonate ring (Scheme 3). VCC 1k (incorporating a spiro-fused cyclohexyl group) was first chosen and combined with several Hantzsch esters furnishing 3ka-3kg. In these sterically frustrated transformations, the b:I ratios remained in most cases practical allowing to isolate the pure, branched homoallylic alcohols in moderate to good yields (48-72\%). Furthermore, the conversion of $\mathbf{1} \mathbf{k}$ proceeded smoothly with a quantum yield $6.8 \%$ leading to complete conversion within $20 \mathrm{~min}$ (see SI). ${ }^{13}$ Despite the more complex nature of these couplings compared to the ones presented in Scheme 2, slightly higher enantiomeric ratios of up to 92.5:7.5 were noted. Increasing the size of the spiro-cycloalkyl group in the VCC (3la, 3lb and 3ld) was feasible and the protocol was effective towards the formation of chiral homoallylic alcohol products in reasonable yields and with 
er values of up to 94:6. Next, an acyclic substitution on the tetrasubstituted VCC substrates was examined (i.e., $\mathbf{1 m}$ ) delivering the target products $\mathbf{3 m a}$ and $\mathbf{3} \mathbf{m b}$ with slightly higher b:I ratios and isolated yields (for $\mathbf{3 m a}$, 74\%, and $3 \mathrm{mb}, 86 \%)$.

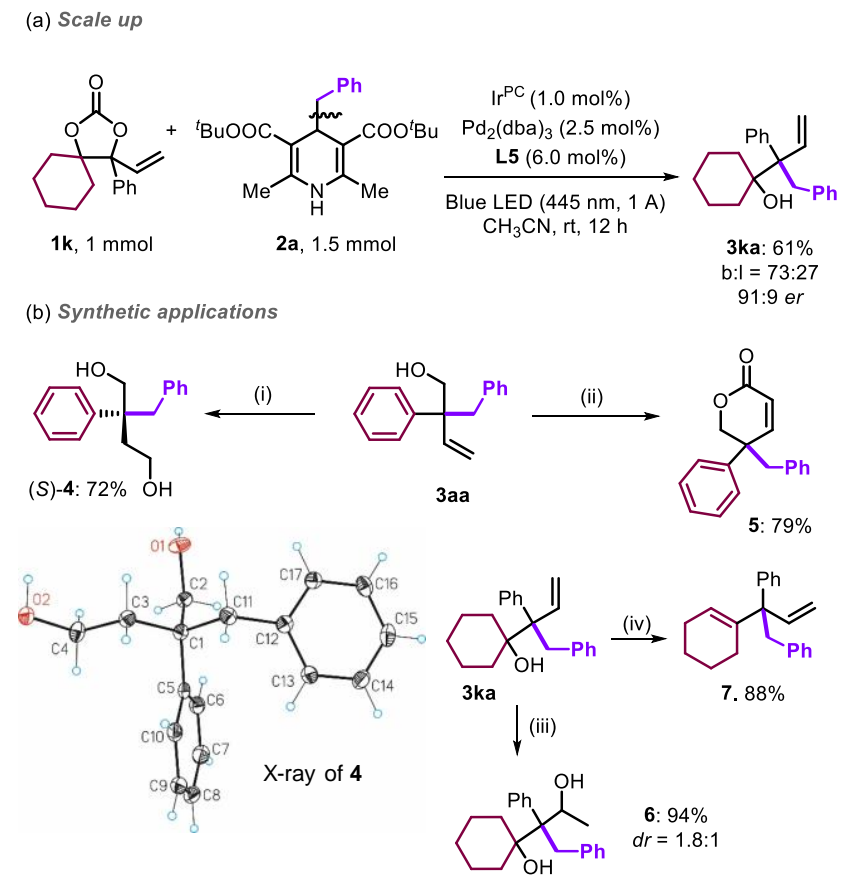

Scheme 4. (a) Scale-up and (b) product diversification. Reaction conditions: (i) $\mathrm{BH}_{3} \cdot \mathrm{THF}$, dry THF, 0 o $\mathrm{C}, 3 \mathrm{~h}$; then $\mathrm{NaOH}_{2} \mathrm{H}_{2} \mathrm{O}_{2}$. (ii) acryloyl chloride, DIPEA, $\mathrm{CH}_{2} \mathrm{Cl}_{2}, \mathrm{rt}, 2 \mathrm{~h}$; then $\mathrm{H}_{2} \mathrm{O}+$ work up; then $2^{\text {nd }}$ generation Hoveyda-Grubbs cat. (10 mol\%), dry toluene, 80 으, $24 \mathrm{~h}$. (iii) same

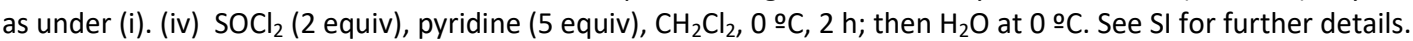

We also studied the use of a trisubstituted VCC (1n). Despite the excellent regioselectivity, the enantiocontrol was significantly lower while producing the product with low diastereocontrol. ${ }^{14}$ Finally, we used Hantzsch esters that would produce secondary radicals under the experimental conditions, leading to vicinal quaternary-tertiary carbons through a different route. In all these cases (3ah, 3ai and 3aj), the products were formed with high regiocontrol and er values adding further diversity to the developed methodology. The absolute configuration of the major enantiomer of compound 3ma was determined to be $(R)$ by X-ray diffraction. ${ }^{15}$

The synthesis of homoallylic alcohol $3 \mathbf{k a}$ could be conveniently scaled up (10-fold) as shown in Scheme $4 a$. We then used 3aa and 3ka for product diversification studies (Scheme 4b). Hydroboration/oxidation of 3aa provided access to 1,4-diol 4 in 72\%; X-ray analysis revealed that the absolute configuration of the major enantiomer was (S). ${ }^{15}$ A metathesis/cyclization of $\mathbf{3 a a}$ in the presence of acryloyl chloride gave unsaturated lactone $\mathbf{5}$ (79\%). Under similar reaction conditions as for $\mathbf{4}$, the hydroboration/oxidation of 3ka gave a Markovnikov type product (1,3diol $6,94 \%)^{16}$ as an approximate 2:1 mixture of diastereoisomers. Dehydration of 3ka using thionyl chloride produced 1,4-diene 7 in $88 \%$ yield.

Conclusion: In summary, we here describe a Pd-mediated dual catalysis approach that allows for transformation of previously unreactive VCCs into chiral homoallylic alcohols featuring vicinal, highly congested carbon atoms. The developed protocol combines an atypical preference for the formation of branched regioisomers in a 
sterically challenging allylic substitution event, and produces the products with enantiomeric ratios of up to 94:6. The present results mark a significant step forward in the use of modular VCCs in challenging enantioselective syntheses.

\section{Acknowledgments.}

We thank the Cerca program/Generalitat de Catalunya, ICREA, MINECO (CTQ2017-88920-P), AGAUR (2017-SGR232) and the Ministerio de Ciencia e Innovación (Severo Ochoa Excellence Accreditation 2020-2023 CEX2019000925-S) for support. BL thanks the European Community for a Marie Curie individual fellowship (PHOTOCARBOX grant agreement 889754), and DG appreciates the postdoctoral funding from AGAUR (2018-BP00243).

\section{References}

(1) (a) Long, R.; Huang, J.; Gong, J.; Yang, Z. Direct Construction of Vicinal All-Carbon Quaternary Stereocenters in Natural Product Synthesis. Nat. Prod. Rep. 2015, 32, 1584-1601. (b) Büschleb, M.; Dorich, S.; Hanessian, S.; Tao, D.; Schenthal, K. B.; Overman, L. E. Synthetic Strategies Toward Natural Products Containing Contiguous Stereogenic Quaternary Carbon Atoms. Angew. Chem. Int. Ed. 2016, 55, 4156-4186. (c) Liu, Y.; Han, S.-J.; Liu, W.-B.; Stoltz, B. M. Catalytic Enantioselective Construction of Quaternary Stereocenters: Assembly of Key Building Blocks for the Synthesis of Biologically Active Molecules. Acc. Chem. Res. 2015, 48, 740-751. (d) Enquist J. A. Jr; Stoltz, B. M. The total Synthesis of (-)-Cyanthiwigin F by means of Double Catalytic Enantioselective Alkylation. Nature 2008, 453, 1228-1231.

(2) (a) Trost, B. M.; Schultz, J. E. Palladium-Catalyzed Asymmetric Allylic Alkylation Strategies for the Synthesis of Acyclic Tetrasubstituted Stereocenters. Synthesis 2019, 51, 1-30. (b) Süsse, L.; Stoltz, B. M. Enantioselective Formation of Quaternary Centers by Allylic Alkylation with First-Row Transition-Metal Catalysts. Chem. Rev. 2021, DOI: 10.1021/acs.chemrev.0c01115. (c) Douglas, C. J.; Overman, L. E. Catalytic Asymmetric Synthesis of All-Carbon Quaternary Stereocenters. Proc. Natl. Acad. Sci. U. S. A. 2004, 101, 5363-5367. (d) Quasdorf, K. W.; Overman, L. E. Catalytic Enantioselective Synthesis of Quaternary Carbon Stereocenters. Nature 2014, 516, 181-191. (e) Marek, I.; Minko, Y.; Pasco, M.; Mejuch, T.; Gilboa, N.; Chechik, H.; Das, J. P. All-Carbon Quaternary Stereogenic Centers in Acyclic Systems through the Creation of Several C-C Bonds per Chemical Step. J. Am. Chem. Soc. 2014, 136, 2682-2694. (f) Zeng, X.-P.; Cao, Z.-Y.; Wang, Y.-H.; Zhou, F.; Zhou, J. Catalytic Enantioselective Desymmetrization Reactions to All-Carbon Quaternary Stereocenters. Chem. Rev. 2016, 116, 7330-7396. (g) Feng, J.; Holmes, M.; Krische, M. J. Acyclic Quaternary Carbon Stereocenters via Enantioselective Transition Metal Catalysis. Chem. Rev. 2017, 117, 12564-12580.

(3) (a) Pierrot, D.; Marek, I. Synthesis of Enantioenriched Vicinal Tertiary and Quaternary Carbon Stereogenic Centers within an Acyclic Chain. Angew. Chem. Int. Ed. 2020, 59, 36-49. (b) Peterson, E. A.; Overman, L. E. Contiguous Stereogenic Quaternary Carbons: A Daunting Challenge in Natural Products Synthesis. Proc. Natl. Acad. Sci. U. S. A. 2004, 101, 11943-11948. (c) Zhou, F.; Zhu, L.; Pan, B.; Shi, Y.; Liu, Y.; Zhou, J. Catalytic Enantioselective Construction of Vicinal Quaternary Carbon Stereocenters. Chem. Sci. 2020, 11, 9341-9365. For a recent example: (d) Isomura, M.; Petrone, D. A.; Carreira, E. M. Construction of Vicinal Quaternary Centers via Iridium-Catalyzed Asymmetric Allenylic Alkylation of Racemic Tertiary Alcohols. J. Am. Chem. Soc. 2021, 143, 3323-3329.

(4) (a) Cherney, A. H.; Kadunce, N. T.; Reisman, S. E. Enantioselective and Enantiospecific Transition-Metal-Catalyzed Cross-Coupling Reactions of Organometallic Reagents To Construct C-C Bonds. Chem. Rev. 2015, 115, 9587-9652. (b) Pàmies, O.; Margalef, J.; Cañellas, S.; James, J.; Judge, E.; Guiry, P. J.; Moberg, C.; Bäckvall, J.-E.; Pfaltz, A.; Pericàs, M. A.; Diéguez, M. Recent Advances in Enantioselective PdCatalyzed Allylic Substitution: From Design to Applications. Chem. Rev. 2021, DOI: 10.1021/acs.chemrev.0c00736. (c) Huang, H.-M.; Bellotti, P.; Glorius, F. Transition Metal-Catalysed Allylic Functionalization Reactions involving Radicals. Chem. Soc. Rev. 2020, 49, 6186-6197.

(5) (a) Trost, B. M.; Schroeder, G. M. Palladium-Catalyzed Asymmetric Alkylation of Ketone Enolates. J. Am. Chem. Soc. 1999, 121, 67596760. (b) Trost, B. M.; Malhotra, S.; Chan, W. H. Exercising Regiocontrol in Palladium-Catalyzed Asymmetric Prenylations and Geranylation: Unifying Strategy toward Flustramines A and B. J. Am. Chem. Soc. 2011, 133, 7328-7331. (c) Hethcox, J. C.; Shockley, S. E.; Stoltz, B. M. Iridium-Catalyzed Diastereo-, Enantio-, and Regioselective Allylic Alkylation with Prochiral Enolates. ACS Catal. 2016, 6, 6207-6213. (d) Sun, M.; Chen, J.-F.; Chen, S.; Li, C. Construction of Vicinal Quaternary Carbon Centers via Cobalt-Catalyzed Asymmetric Reverse Prenylation. Org. Lett. 2019, 21, 1278-1282. (e) Khan, A.; Yang, L.; Xu, J.; Jin, L. Y.; Zhang, Y. J. Palladium-Catalyzed Asymmetric Decarboxylative Cycloaddition of Vinylethylene Carbonates with Michael Acceptors: Construction of Vicinal Quaternary Stereocenters. Angew. Chem. Int. Ed. 2014, 53, 11257- 11260 .

(6) (a) Hou, X.-L.; Sun, N. Construction of Chiral Quaternary Carbon Centers by Pd-Catalyzed Asymmetric Allylic Substitution with P,N-1,1'Ferrocene Ligands. Org. Lett. 2004, 6, 4399-4401. (b) Zhang, P.; Le, H.; Kyne, R. E.; Morken, J. P. Enantioselective Construction of All-Carbon Quaternary Centers by Branch-Selective Pd-Catalyzed Allyl-Allyl Cross-Coupling. J. Am. Chem. Soc. 2011, 133, 9716-9719. (c) Shockley, S. 
E.; Caleb Hethcox, J. C.; Stolz, B. M. Enantioselective Synthesis of Acyclic $\alpha$-Quaternary Carboxylic Acid Derivatives through IridiumCatalyzed Allylic Alkylation. Angew. Chem. Int. Ed. 2017, 56, 11545-11548. (d) Caleb Hethcox, J. C.; Shockley, S. E.; Stoltz, B. M. Angew. Chem. Int. Ed. 2018, 57, 8664-8667.

(7) (a) Trost, B. M.; McEachern, E. J.; Toste, F. D. A Two-Component Catalyst System for Asymmetric Allylic Alkylations with Alcohol Pronucleophiles. J. Am. Chem. Soc. 1998, 120, 12702-12703. (b) Trost, B. M.; Bunt, R. C.; Lemoine, R. C.; Calkins, T. L. Dynamic Kinetic Asymmetric Transformation of Diene Monoepoxides: A Practical Asymmetric Synthesis of Vinylglycinol, Vigabatrin, and Ethambutol. J. Am. Chem. Soc. 2000, 122, 5968-5976. (c) Trost, B. M.; Jiang, C. Atom Economic Asymmetric Creation of Quaternary Carbon: Regio- and Enantioselective Reactions of a Vinylepoxide with a Carbon Nucleophile. J. Am. Chem. Soc. 2001, 123, 12907-12908. (d) Cheng, Q.; Zhang, H.-J.; You, S.-L. Palladium-Catalyzed Highly Stereoselective Dearomative [3+2] Cycloaddition of Nitrobenzofurans. Chem 2017, 3, 428-436. (e) Feng, J.; Garza, V. J.; Krische, M. J. Redox-Triggered C-C Coupling of Alcohols and Vinyl Epoxides: Diastereo- and Enantioselective Formation of All-Carbon Quaternary Centers via tert-(Hydroxy)-Prenylation. J. Am. Chem. Soc. 2014, 136, 8911-8914.

(8) (a) Cai, A.; Guo, W.; Martínez-Rodríguez, L.; Kleij, A. W. Palladium-Catalyzed Regio- and Enantioselective Synthesis of Allylic Amines Featuring Tetrasubstituted Tertiary Carbons. J. Am. Chem. Soc. 2016, 138, 14194-14197. (b) Guo, W.; Cai, A.; Xie, J.; Kleij, A. W. Asymmetric Synthesis of alfa,alfa-Disubstituted Allylic Amines via Pd-Catalyzed Allylic Substitution. Angew. Chem. Int. Ed. 2017, 56, 11797-11801. (c) Cai, A.; Kleij, A. W. Regio- and Enantioselective Preparation of Chiral Allylic Sulfones Featuring Elusive Quaternary Stereocenters. Angew. Chem. Int. Ed. 2019, 58, 14944-14949. (d) Khan, A.; Zhao, H.; Zhang, M.; Khan, S.; Zhao, D. Regio- and Enantioselective Synthesis of SulfoneBearing Quaternary Carbon Stereocenters by Pd-Catalyzed Allylic Substitution. Angew. Chem. Int. Ed. 2020, 59, 1340-1345. (e) Khan, A.; Khan, S.; Khan, I.; Zhao, C.; Mao, Y.; Chen, Y.; Zhang, Y. J. Enantioselective Construction of Tertiary C-O Bond via Allylic Substitution of Vinylethylene Carbonates with Water and Alcohols. J. Am. Chem. Soc. 2017, 139, 10733-10741. (f) Khan, A.; Xing, J.; Zhao, J.; Kan, Y.; Zhang, W.; Zhang, Y. J. Palladium-Catalyzed Enantioselective Decarboxylative Cycloaddition of Vinylethylene Carbonates with Isocyanates. Chem. Eur. J. 2015, 21, 120-124.

(9) (a) Cristòfol, À.; Böhmer, C.; Kleij, A. W. Formal Synthesis of Indolizidine and Quinolizidine Alkaloids from Vinyl Cyclic Carbonates. J. Org. Chem. 2018, 83, 9978-9990. (b) Ke, M.; Liu, Z.; Huang, G.; Wang, J.; Tao, Y.; Chen, F. Palladium-Catalyzed Regio- and Stereoselective CrossCoupling of Vinylethylene Carbonates with Ketimine Esters to Generate (Z)-Tri- and Tetra-substituted Allylic Amino Acid Derivatives. Org. Lett. 2020, 22, 4135-4140. (c) Wang, Y.; Chai, J.; You, C.; Zhang, J.; Mi, X.; Zhang, L.; Luo, S. $\pi$-Coordinating Chiral Primary Amine/Palladium Synergistic Catalysis for Asymmetric Allylic Alkylation. J. Am. Chem. Soc. 2020, 142, 3184-3195; d) Wei, X.; Liu, D.; An, Q.; Zhang, W. Hydrogen-Bond Directed Regioselective Pd-Catalyzed Asymmetric Allylic Alkylation: The Construction of Chiral $\alpha$-Amino Acids with Vicinal Tertiary and Quaternary Stereocenters. Org. Lett. 2015, 17, 5768-5771. (e) Guo, W.; Martínez-Rodríguez, L.; Kuniyil, R.; Martin, E.; Escudero-Adán, E. C.; Maseras, F.; Kleij, A. W. Stereoselective and Versatile Preparation of Tri- and Tetrasubstituted Allylic Amine Scaffolds under Mild Conditions. J. Am. Chem. Soc. 2016, 138, 11970-11978. (f) Chen, L.; Quan, H.; Xu, Z.; Wang, H.; Xia, Y.; Lou, L.; Yang, W. A Modular Biomimetic Strategy for the Synthesis of Macrolide P-Glycoprotein Inhibitors via Rh-Catalyzed C-H Activation. Nat. Commun. 2020, 11, 2151. (g) Deng, L.; Kleij, A. W.; Yang, W. Diversity-Orientated Stereoselective Synthesis through Pd-Catalyzed Switchable Decarboxylative C-N/C-S Bond Formation in Allylic Surrogates. Chem. Eur. J. 2018, 24, 19156-19161. (h) Wang, H.; Qiu, S.; Wang, S.; Zhai, H. Pd-Catalyzed Umpolung of $\pi$-Allylpalladium Intermediates: Assembly of All-Carbon $\alpha$-Vinyl Quaternary Aldehydes through $C\left(s p^{3}\right)-C\left(s p^{3}\right)$ Coupling. ACS Catal. 2018, 8, 11960-11965. (i) Guo, W.; Kuniyil, R.; Gómez, J. E.; Maseras, F.; Kleij, A. W. A Domino Process towards Functionally Dense Quaternary Carbons through Pd-Catalyzed Decarboxylative Csp ${ }^{3}-\mathrm{Csp}^{3}$ Bond Formation. J. Am. Chem. Soc. 2018, 140, 3981-3987.

(10) (a) Lang, S. B.; O’Nele, K. M.; Tunge, J. A. Decarboxylative Allylation of Amino Alkanoic Acids and Esters via Dual Catalysis. J. Am. Chem. Soc. 2014, 136, 13606-13609. (b) Lang, S. B.; O'Nele, K. M.; Douglas, J. T.; Tunge, J. A. Dual Catalytic Decarboxylative Allylations of $\alpha-A m i n o$ Acids and Their Divergent Mechanisms. Chem. Eur. J. 2015, 21, 18589-18593. (c) Xuan, J.; Zeng, T.-T.; Feng, Z.-J.; Deng, Q.-H.; Chen, J.-R.; Lu, L.-Q.; Xiao, W.-J.; Alper, H. Redox-Neutral $\alpha$-Allylation of Amines by Combining Palladium Catalysis and Visible-Light Photoredox Catalysis. Angew. Chem. Int. Ed. 2015, 54, 1625-1628. (d) Matsui, J. K.; Gutiérrez-Bonet, Á.; Rotella, M.; Alam, R.; Gutierrez, O.; Molander, G. A. Photoredox/Nickel-Catalyzed Single-Electron Tsuji-Trost Reaction: Development and Mechanistic Insights. Angew. Chem. Int. Ed. 2018, 57, 15847-15851. (e) Ye, H.; Ye, Q.; Cheng, D.; Li, X.; Xu, X. The Coupling of Potassium Organotrifluoroborates with Baylis-Hillman Derivatives via Visible-Light Photoredox Catalysis. Tetrahedron Lett. 2018, 59, 2046-2049. (f) Zhang, H.-H.; Zhao, J.-J.; Yu, S. Enantioselective Allylic Alkylation with 4-Alkyl-1,4-dihydro-pyridines Enabled by Photoredox/Palladium Cocatalysis. J. Am. Chem. Soc. 2018, 140, 1691416919. (g) Zhang, H.-H.; Zhao, J.-J.; Yu, S. Enantioselective $\alpha$-Allylation of Anilines Enabled by a Combined Palladium and Photoredox Catalytic System. ACS Catal. 2020, 10, 4710-4716. (h) Luca Schwarz, J.; Huang, H.-M.; Paulisch, T. O.; Glorius, F. Dialkylation of 1,3-Dienes by Dual Photoredox and Chromium Catalysis. ACS Catal. 2020, 10, 1621-1627. (i) Li, F.-S.; Chen, Y.-Q.; Lin, S.-J.; Shi, C.-Z.; Li, X.-Y.; Sun, Y.C.; Guo, Z.-W.; Shi, L. Visible-Light-Mediated Barbier Allylation of Aldehydes and Ketones via Dual Titanium and Photoredox Catalysis. Org. Chem. Front. 2020, 7, 3434-3438. (j) Gualandi, A.; Rodeghiero, G.; Perciaccante, R.; Jansen, T. P.; Moreno-Cabrerizo, C.; Foucher, C.; Marchini, M.; Ceroni, P.; Cozzi, P. G. Catalytic Photoredox Allylation of Aldehydes Promoted by a Cobalt Complex. Adv. Synth. Catal. 2021, 363, 1105-1111. (k) Gualandi, A.; Calogero, F.; Mazzarini, M.; Guazzi, S.; Fermi, A.; Bergamini, G.; Cozzi, P. G. Cp PTiCl $_{2}$-Catalyzed Photoredox Allylation of Aldehydes with Visible Light. ACS Catal. 2020, 10, 3857-3863. (I) Schwarz, J. L.; Schäfers, F.; Tlahuext-Aca, A.; Lückemeier, L.; Glorius, F. Diastereoselective Allylation of Aldehydes by Dual Photoredox and Chromium Catalysis. J. Am. Chem. Soc. 2018, 140, 1270512709. (m) Twilton, J.; Le, C.; Zhang, P.; Shaw, M. H.; Evans, R. W.; MacMillan, D. W. C. The Merger of Transition Metal and Photocatalysis. Nat. Rev. Chem. 2017, 1, 0052. 
(11) (a) Crespi, S.; Fagnoni, M. Generation of Alkyl Radicals: From the Tyranny of Tin to the Photon Democracy. Chem. Rev. 2020, 120, 97909833. (b) Huang, W.; Cheng, X. Hantzsch Esters as Multifunctional Reagents in Visible-Light Photoredox Catalysis. Synlett. 2017, 28, 148158. (c) Matsui, J. K.; Lang, S. B.; Heitz, D. R.; Molander, G. A. Photoredox-Mediated Routes to Radicals: The Value of Catalytic Radical Generation in Synthetic Methods Development. ACS Catal. 2017, 7, 2563-2575.

(12) (a) Huang, X.; Luo, S.; Burghaus, O.; Webster, R. D.; Harms, K.; Meggers. E. Combining the Catalytic Enantioselective Reaction of VisibleLight-generated Radicals with a By-Product Utilization System. Chem. Sci. 2017, 8, 7126-7131. (b) Buzzetti, L.; Prieto, A.; Roy, S. R.; Melchiorre, P. Radical-Based C-C Bond-Forming Processes Enabled by the Photoexcitation of 4-Alkyl-1,4-dihydropyridines. Angew. Chem. Int. Ed. 2017, 56, 15039-15043.

(13) The oxidation of $2 \mathrm{a}$ to its corresponding pyridine proceeds with a quantum yield of $7.5 \%$, indicating that the coupling reaction proceeds immediately once the alkyl radicals are released using $>90 \%$ of the radicals productively.

(14) Low diastereocontrol is a general phenomenon in the creation of vicinal quaternary/tetrasubstituted carbon stereocenters, see for instance ref. 3a and 3d.

(15) This configuration was determined for several crystals that were independently analyzed by X-ray diffraction, see the SI for details.

(16) The discrepancy between the hydroboration/oxidation of 3aa and 3ka is suggested to arise from a directing-group effect of the conformationally more restricted tertiary $\mathrm{OH}$ in $\mathbf{3 k a}$. 
